\title{
Clinical Course of Five COVID-19 Patients and Treatment Updates
}

\author{
Mohsin Sheraz Mughal ${ }^{1,}$, , Ikwinder Preet Kaur ${ }^{1}, \operatorname{Rameez}_{\operatorname{Rehman}}{ }^{1}, \operatorname{Hasan}_{\text {Mahmood Mirza }}{ }^{2}$, \\ Harjot Singh Jagdey ${ }^{3}$, Chandler Patton ${ }^{1}$, Wael Ghali ${ }^{1}$ \\ ${ }^{1}$ Monmouth Medical Center, Long Branch, New Jersey, United States \\ ${ }^{2}$ Harvard Medical School, Beth Israel Deaconess Medical Center, Boston, United States \\ ${ }^{3}$ BronxCare Health System, Bronx, United States
}

Email address:

mohsin.sheraz.mughal@gmail.com (M. S. Mughal)

${ }^{*}$ Corresponding author

\section{To cite this article:}

Mohsin Sheraz Mughal, Ikwinder Preet Kaur, Rameez Rehman, Hasan Mahmood Mirza, Harjot Singh Jagdey, Chandler Patton, Wael Ghali. Clinical Course of Five COVID-19 Patients and Treatment Updates. International Journal of Infectious Diseases and Therapy. Vol. 5, No. 3, 2020, pp. 74-80. doi: 10.11648/j.ijidt.20200503.17

Received: July 10, 2020; Accepted: July 28, 2020; Published: August 20, 2020

\begin{abstract}
Coronaviruses (CoVs) are enveloped, positive-sense RNA viruses. A novel coronavirus (nCoV) was discovered to be a cause of pneumonia outbreak in Wuhan, China in late 2019. The World Health Organization (WHO) named the disease as Coronavirus Disease 2019 (COVID-19) and declared it as a pandemic in March 2020. Since the detection of the first case of COVID-19 in the United States on January 20th, 2020 in Washington State, the pandemic is still expanding. As of July 8th, the United States (U.S.) has the maximum number (3.1 million) of confirmed COVID-19 patients. In this case series, we are presenting the clinical course of the first five confirmed COVID-19 cases admitted to a community hospital in the U.S. in the beginning of March 2020. We analyzed their clinical characteristics, pre-existing comorbidities, laboratory and radiological findings. The clinical management and outcomes are discussed alongside the literature review about current management options. In conclusion, primary management is supportive care to improve oxygenation by various methods. Safety and efficacy of treatment options including ramdesevir, IL-6 inhibitors and convalescent plasma have not yet been established and none of these is FDA approved so far. Increased age ( $>80$ years), hypoxia at the time of presentation and pre-existing comorbidities are likely related with poor outcomes.
\end{abstract}

Keywords: Pneumonia, Coronavirus, Pandemic

\section{Introduction}

Late 2019, a novel coronavirus (nCoV) was implicated for a cluster of pneumonia outbreak in Wuhan, China. The International Committee on Taxonomy of Viruses (ICTV) named it Severe Acute Respiratory Syndrome Coronavirus 2 (SARS-CoV-2) [1]. The World Health Organization (WHO) named the disease as Coronavirus Disease 2019 (COVID-19) and declared coronavirus disease 2019 (COVID-19) a pandemic in March 2020. As of July 8th, the U.S has the maximum number (3.1 million) of confirmed COVID-19 patients [2]. Initial studies from China provided a basic understanding of the epidemiology of the disease, demographics of the population, radiological evidence of pneumonia, and its human to human transmission. While the pandemic continues to spread, more information is available imparting a better understanding of the disease process, its presentation, and related complications. Different parameters like age, pre-existing comorbidities, and deranged inflammatory markers play a significant role in the severity of the disease and may lead to worse outcomes in a certain population subset [3]. In this manuscript, we will discuss the demographic characteristics, pre-existing comorbidities, clinical manifestations, disease markers, clinical course and the treatment in five different cases admitted at the beginning of pandemic to a community hospital along with literature review. In this review, we discussed the updated management of COVID-19 and success of different therapeutic trials. 


\section{Methods}

This descriptive study constitutes a case series of five confirmed adult COVID-19 cases. Patients were admitted to the community hospital in the United States at the beginning of March 2020. Cases were confirmed using real-time Reverse Transcriptase-Polymerase Chain Reaction (RT-PCR) assay of nasopharyngeal swabs. Clinical data were collected using electronic medical records. An extensive literature review was performed using medical search databases such as PubMed and online medical journals.

\section{Cases Description}

\section{Case 1}

The patient is a 39-year-old obese male who presented with the complaints of persistent fever, progressive dry cough, generalized body aches and excessive sleep for the last five days. His medical problems included hypertension (HTN), gastroesophageal reflux disease (GERD), arthritis, opioid abuse and recurrent cellulitis. Patient's medication list included lisinopril, esomeprazole and suboxone. He didn't mention any known contact with COVID-19 positive cases, though he used to commute to a different city on a train. The patient presented to the Emergency Department (ED) urgent care two days before this admission and was prescribed azithromycin for fever and cough without any relief. On examination, the patient was hypertensive and had a lowgrade fever. Chest examination was positive for rhonchi throughout the lung fields. Otherwise, the examination was unremarkable. He was saturating in low 90s with 2-4 L of supplemental $\mathrm{O}_{2}$ via nasal cannula throughout the hospitalization. Laboratory data on admission are shown in table 1. The chest X-Ray (CXR) showed multifocal patchy airspace opacities throughout the lungs, compared to CXR four days back which was normal (Figure 1). CT chest showed innumerable subcentimeter scattered patchy nodules throughout the lungs, more prominent in the peripheral and basilar aspects. (Figure 2)
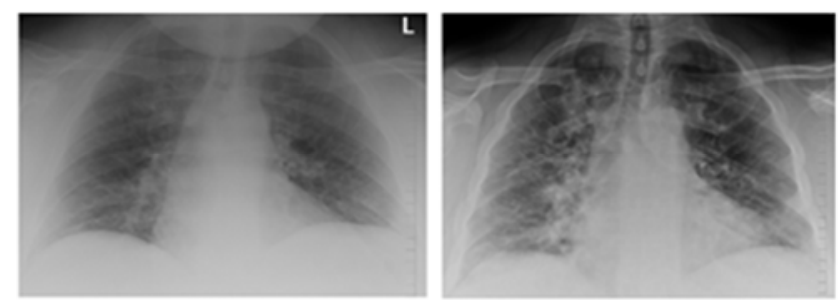

Figure 1. AP chest x-ray from admission (left) shows no evidence of pulmonary infiltrates. Repeat $x$-ray three days later (right) shows new multifocal airspace opacities bilaterally.

The patient was managed with all the precautions for COVID-19 as per hospital protocol and hydroxychloroquine for five days. He was treated with mupirocin nasal ointment for positive MRSA nares. The patient clinically did well, saturated above $90 \%$ on room air and was discharged home after five days of hospitalization. The patient was instructed to remain quarantined for 2 weeks at his home.

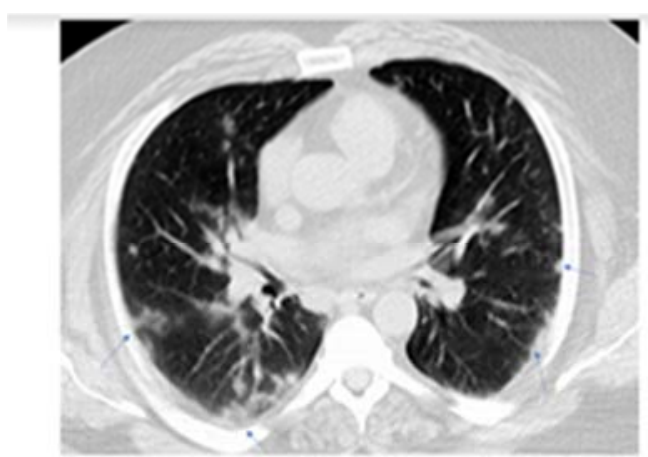

Figure 2. Non-contrast axial CT chest shows multifocal patchy, peripheral nodular opacities.

Case 2

The patient is a 73-year-old female presented from a rehabilitation facility with the complaints of fever, chills, nausea on the day of admission along with generalized weakness and poor appetite. Medical/surgical history included bilateral total knee arthroplasty, HTN, rheumatoid arthritis, Myocardial Infarction (MI), recent Pulmonary Embolism (PE) three weeks back and right knee Purulent Joint Infection (PJI). Patient's medication list included amlodipine, omeprazole, rivaroxaban, Claritin, fish oil and Mucinex. For PJI, initial synovial fluid culture was positive for Staphylococcus Lugdunensis. She was receiving intravenous cefazolin and oral rifampin for PJI. On examination, the patient was hypertensive and febrile with a temperature of $102.7 \mathrm{~F}$ in the ED. Chest examination was unremarkable on the day of admission, however evolved to sporadic rhonchi later on. Right knee was warm, non-tender and without swelling. Otherwise, the examination was unremarkable. Laboratory data on the admission are shown in table 1. Urine Culture was positive for Enterococcus, for which she was treated with Linezolid. She was also started on oral cefuroxime BID during this hospitalization as suppressive therapy for PJI after completion of six weeks of intravenous therapy. The patient developed cough two days after the admission and remained febrile even on appropriate antibiotics. Her repeat joint fluid cultures were negative. Blood cultures were negative. CXR (Figure 3 left) done on admission was negative for consolidations or pleural effusion. CT scan (Figure 4) of chest done after two days of admission showed multifocal ill-defined regions of subpleural groundglass attenuation and nodular consolidations throughout the lung fields bilaterally. It also showed mild hilar lymphadenopathy prominent on the right. Repeat chest XRay (Figure 3 right) was consistent with CT chest.

In addition to COVID-19 precautions, the patient was started on hydroxychloroquine $400 \mathrm{mg}$ BID on the first day, later $200 \mathrm{mg}$ BID to complete a total of five days. Patient was started on azithromycin for five days as well. The patient exhibited clinical improvement slowly. The temperature started trending down, and she was saturating above $94 \%$ on room air at the time of discharge. CRP level peaked around a week of admission and then trended down. She was discharged after 12 days of hospitalization. 

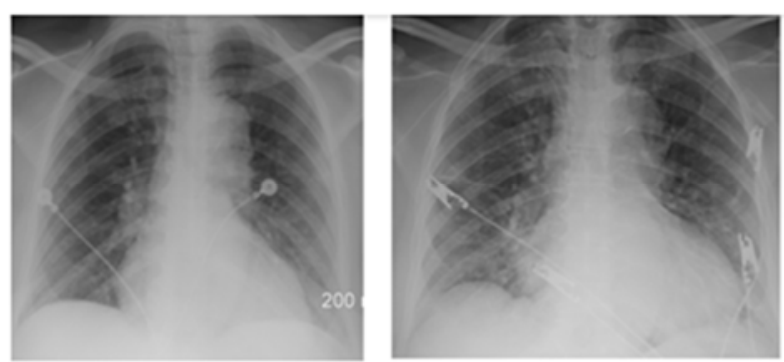

Figure 3. AP chest x-ray from admission (left) shows no evidence of pulmonary consolidations. Repeat $x$-ray five days later (right) shows new ill defined airspace opacities bilaterally, predominantly peripheral in distribution.

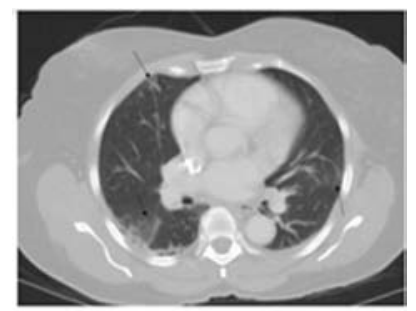

Figure 4. Contrast enhanced chest CT consistent with multiple ill defined subpleural ground glass opacities bilaterally.

\section{Case 3}

The patient is a 60-year-old female with a history of hypertension and came with the complaints of dry cough, fever and progressive dyspnea for a week. She had recent contact with COVID-19 positive patient. She had difficulty in talking because of the cough and SOB. Her medication list included valsartan-HCTZ PO daily. Her primary care physician prescribed azithromycin which didn't help. She also complained of non-bloody watery diarrhea. She was febrile with a temperature of 103.6 on admission. She was saturating $95 \%$ on room air, although she seemed dyspneic and had a frequent cough on examination. Chest auscultation revealed scattered rhonchi in bilateral lung fields. Laboratory data on admission are shown in table 1. Her chest X-Ray (Figure 5 left) showed patchy areas of increased density. She was started on hydroxychloroquine $200 \mathrm{mg}$ BID and azithromycin daily for a total of five days. However, she continued to have fever and cough and on the third day, she desaturated to ' 80 s on nasal cannula requiring a venti mask and later high-flow to maintain O2 sat $>90 \%$. She was upgraded to the ICU level of care. She was started on $\mathrm{Zn}$ which was discontinued due to the development of nausea and vomiting. Loperamide was added for symptomatic treatment of diarrhea (Clostridium difficile test was negative). Her CRP and ferritin peaked after about five days of hospitalization and then started trending down. Repeat CXR (Figure 5 right) done in ICU showed worsening of pulmonary infiltrate. After about a week in the ICU, the patient's breathing was stable, therefore she was downgraded to general medical floors for further care. Patient is still in the hospital and clinically doing well.

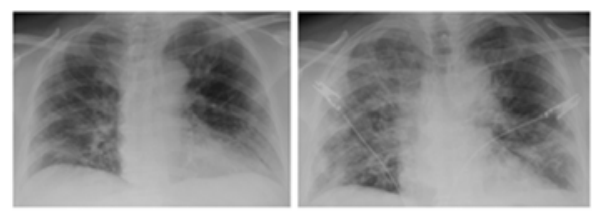

Figure 5. AP chest $x$-ray admission (left) shows patchy areas of increased density along the bilateral lung bases. Repeat $x$-ray eight days later (right) shows worsening persistent pulmonary infiltrates bilaterally, right sided greater than left.

\section{Case 4}

The patient was a 47-year-old health care worker with no past medical history, presented to ED with the complaints of fever, headache, dry cough, poor oral intake for about 10 days. He intubated a patient around two weeks before coming to the hospital, which turned out to be COVID-19 positive. Patient started developing symptoms one day after intubation. He decided to self-quarantine himself. He treated himself with hydration, tylenol and azithromycin. However, a day before admission he started having diarrhea, felt generalized weak, dehydrated and nauseated that prompted him to come to the ED. On examination, vitals were stable and the patient was afebrile. Chest examination was unremarkable. Laboratory data on admission are shown in table 1. CXR (Figure 6) was negative for definitive consolidations. CT scan (Figure 7) showed diffuse ground-glass opacities throughout the lungs with the peripheral distribution.

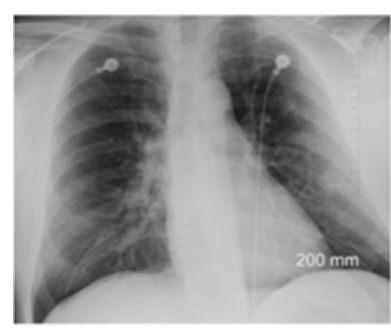

Figure 6. AP chest $x$-ray from admission shows no definite pulmonary consolidations or opacities bilaterally.

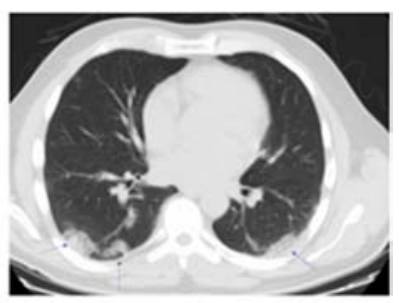

Figure 7. Non-contrast enhanced axial CT of the chest demonstrates diffuse bilateral ground glass opacities with a predominantly peripheral distribution.

He completed five days of hydroxychloroquine and azithromycin. Diarrhea and nausea resolved although the patient continued to have a dry cough. He remained stable and was discharged after four days of hospitalization.

Case 5

The patient is an 83-year-old male came with the complaints of mild chest congestion, dry cough, fever and worsening dyspnea on the day of admission. He had a history of COPD, coronary artery disease, diastolic heart failure, multiple aortic 
aneurysms, chronic kidney disease, HTN, hyperlipidemia, diabetes mellitus, and deep venous thrombosis. He had a recent diagnosis of chronic PE and was discharged from hospital a day before current admission. Patient's medications included metoprolol, amlodipine, atorvastatin, apixaban, omeprazole and various inhalers for COPD. He had exposure to COVID-19 positive people. On examination, vitals were: BP 146/63, HR 108 , RR 28, and temperature 101.6 F. The patient was saturating $92 \%$ on $5 \mathrm{~L}$ of supplemental $\mathrm{O} 2$. Chest examination revealed decreased breath sounds in bilateral lung fields along with wheezing. He was febrile during the previous hospitalization as well. However, considering PE might be the reason, was discharged home as he remained afebrile for a day. CT chest (Figure 8) findings were consistent with emphysematous changes and multifocal regions of sub pleural reticulo-nodular opacities throughout the lungs. Laboratory data on this admission are shown in table 1. CXR was stable (Figure 9 left).

The patient was started on intravenous steroids and supportive management for possible COPD exacerbation. However, because of worsening hypoxia on $5 \mathrm{~L}$ of nasal cannula, he was switched to Hi-flow. Hydroxychloroquine and azithromycin were started empirically at this point before the results came positive. He completed five days of azithromycin and seven days of hydroxychloroquine along with zinc supplementation. After a week, the patient started requiring high $\mathrm{FiO} 2$ and was transferred to the ICU, repeat CXR (Figure 9 right) showed increased interstitial lung markings. The patient remained persistently hypoxic on Hi- flow and a decision was made to intubate him. Over the course of stay, he remained acidotic and hypoxic even after intubation with no improvement in respiratory status. Knowing the poor prognosis, he was terminally extubated.

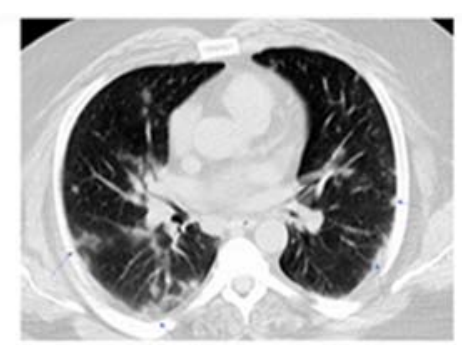

Figure 8. Non-contrast axial CT chest shows multifocal patchy, peripheral nodular opacities.

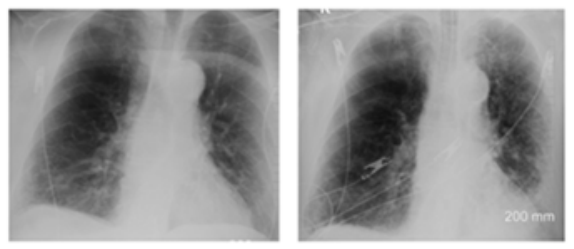

Figure 9. AP-chest $x$-ray from admission (left) shows bibasilar atelectasis without evidence of pulmonary consolidation. Repeat $x$-ray one week later (right) shows increased reticular and interstitial lung markings worse on the left. Incidentally seen is blunting of the bilateral costophrenic angles.

Demographic characteristics, clinical manifestations, disease markers and outcomes of five patients with COVID19 pneumonia (Table 1).

Table 1. Clinical characteristics of COVID-19 patients.

\begin{tabular}{|c|c|c|c|c|c|}
\hline & Case 1 & Case 2 & Case 3 & Case 4 & Case 5 \\
\hline Age (years) & 39 & 73 & 60 & 47 & 83 \\
\hline Sex & M & $\mathrm{F}$ & $\mathrm{F}$ & M & M \\
\hline Contact w COVID-19 confirmed case & No & No & Yes & Yes & Yes \\
\hline \multicolumn{6}{|l|}{ Pre-existing comorbidities } \\
\hline Heart Failure & No & No & No & No & Yes \\
\hline COPD & No & No & No & No & Yes \\
\hline DM & No & No & No & No & Yes \\
\hline Past history of smoking & Yes & Yes & No & No & Yes \\
\hline On ACE Inhibitor/ARBs & Yes & No & Yes & No & No \\
\hline Duration of symptoms before hospital admission (days) & 5 & 1 & 7 & 10 & 1 \\
\hline $\mathrm{O}_{2}$ sat on admission $(\%)$ & 93 on $2 \mathrm{~L}$ & 96 on RA & 95 on RA & 98 on RA & 92 on $5 \mathrm{~L} \mathrm{NC}$ \\
\hline \multicolumn{6}{|l|}{ Laboratory data on admission } \\
\hline WBC count $(4.5-11 \mathrm{k} / \mathrm{cmm})$ & 3 & 3 & 3.5 & 8.4 & 6.6 \\
\hline Lymphocyte count $(20-45 \%)$ & 21 & 28 & 13 & 13 & 7 \\
\hline Absolute N: L ratio (NLR-0.78-3.53) & 3.5 & 2.1 & 5.6 & 5.8 & 11.4 \\
\hline Platelet count $(140-450 \mathrm{k} / \mathrm{cmm})$ & 137 & 150 & 183 & 212 & 104 \\
\hline $\operatorname{ESR}(0-30 \mathrm{~mm} / \mathrm{hr})$ & N/A & 74 & N/A & N/A & N/A \\
\hline $\mathrm{CRP}(<7 \mathrm{mg} / \mathrm{L})$ & 49.6 & 66.8 & 96.9 & 8.2 & 54.9 \\
\hline LDH (116-243 IU/L) & N/A & N/A & 524 & Normal & 286 \\
\hline LFT & Normal & Normal & Raised & Normal & Normal \\
\hline Ferritin $(10-291 \mathrm{ng} / \mathrm{ml})$ & N/A & N/A & 1014 & 330 & 230 \\
\hline IL-6 $(<5 \mathrm{pg} / \mathrm{ml})$ & N/A & N/A & 71.78 & N/A & $\mathrm{N} / \mathrm{A}$ \\
\hline Procalcitonin $(<0.50 \mathrm{ng} / \mathrm{ml})$ & Normal & N/A & Normal & Normal & Normal \\
\hline D Dimer & N/A & N/A & $\mathrm{N} / \mathrm{A}$ & N/A & High \\
\hline Nasal MRSA & Positive & N/A & Negative & N/A & Negative \\
\hline Respiratory pathogen panel PCR & Negative & Negative & Negative & N/A & Negative \\
\hline
\end{tabular}




\begin{tabular}{|c|c|c|c|c|c|}
\hline & Case 1 & Case 2 & Case 3 & Case 4 & Case 5 \\
\hline POCT Flu A, B & Negative & Negative & Negative & N/A & Negative \\
\hline Blood culture & Negative & Negative & Negative & N/A & Negative \\
\hline Lower Respiratory culture & Negative & Negative & Negative & N/A & Negative \\
\hline Drugs for treatment & $\mathrm{H}$ & $\mathrm{H}, \mathrm{A}$ & $\mathrm{H}, \mathrm{A}$ & $\mathrm{H}, \mathrm{A}$ & $\mathrm{H}, \mathrm{A}, \mathrm{Z}$ \\
\hline Duration of treatment (Days) & 5 & 5 & 7 & 5 & 7 \\
\hline Type of supplemental O2 used & & & & None & \\
\hline NC (Liter) & Yes, maximum 4L & Maximum $3 \mathrm{~L}$ & Yes & No & Yes \\
\hline Venti Mask & No & No & Yes & No & No \\
\hline Hi Flo & No & No & Yes & No & Yes \\
\hline Ventilator/Intubated & No & No & No & No & Yes \\
\hline ICU level of care & No & No & Yes & No & Yes \\
\hline Outcome & Discharged & Discharged & In hospital & Discharged & Died \\
\hline Length of hospital stay (days) & 5 & 12 & 10 & 4 & 9 \\
\hline Readmission in 2 weeks & No & No & & No & \\
\hline
\end{tabular}

A. Respiratory pathogen panel PCR included Influenza, Parainfluenza, RSV, Coronavirus, Adenovirus, Rhinovirus, Bordetella pertussis, Chlamydophila, Mycoplasma.

B. Drugs for treatment includes Hydroxychloroquine (H), Azithromycin (A) and Zinc (Z).

\section{Discussion}

Since the declaration of COVID-19 pandemic, the whole world is locked down to prevent the community spread and rising death toll. Amid fear, humanity is fighting this deadly virus valiantly. The primary source of transmission of SARS$\mathrm{CoV} 2$ is droplets (particles 5-10um in size). Other sources can be aerosols (particulate size less than 5um), especially during aerosol-generating procedures including cardiopulmonary resuscitation, endotracheal intubation, suctioning, turning the patient to the prone position etc. Human to human transmission can occur either through direct contact with an infected person who emits virus particles while coughing, sneezing, and talking or when another person comes in contact with fomites [4].

The course of the disease can vary from asymptomatic infections to severe pneumonia and multi-organ failure. A study suggested fever (99\%), malaise (70\%) and dry cough $(59 \%)$ as the most common presenting symptoms [5]. So far, RT-PCR has been used as a primary method of detecting SARS-CoV-2 in the clinical setting. rRT PCR is a qualitative detection of SARS CoV-2 RNA nucleic acid in the respiratory tract. However, the actual sensitivity and specificity of the test are not known, also inadequate sampling can affect the test results. The nasopharyngeal swabs have high rates of detecting SARS-CoV-2 compared to oropharyngeal swabs. Importantly positive rRT PCR does not rule out the possibility of other infections and should not be used as a sole decision-maker to rule in COVID-19, especially in the setting of high clinical suspicion. If rRT PCR via nasopharyngeal swab is negative, and there is clinical suspicion, re-sampling from a different respiratory location is recommended. People who have a recent travel history to an endemic area, or have been exposed to someone diagnosed with COVID-19 within fourteen days, or have the symptoms are being screened by real-time reverse transcriptase-polymerase chain reaction (rRT PCR) with nasopharyngeal swabs [6]. Besides, chest CT scans and IgM ELISA can significantly aid in the diagnostic workup. Chest computed tomography has emerged as an integral part of the diagnosis alongside the rRT CPR. Ground-glass opacities were observed in up to $88 \%$ of the patients and bilateral involvement was seen in up to $87.5 \%$. In another study, CT manifestations reported peripheral airspace consolidations in one-third of the cases. Less common findings included pleural effusion, pericardial effusion, cavitation, and pneumothorax. Cases have been reported when initial rRT PCR was negative but CT scan findings were suggestive of COVID-19, later they came positive for SARS-CoV-2. CT's findings can vary upon the day of imaging and some studies even suggested doing a repeat CT scan to rule out the worsening of the disease [7-10].

Once diagnosed, it is very important to categorize the severity of illness, a report from the Chinese CDC suggested $81 \%$ of the infected COVID-19 patients have mild or no pneumonia, $14 \%$ have the severe disease as they have hypoxia or radiological evidence of $50 \%$ or more involvement of lung area. Critically ill patients are those who have respiratory failure, shock or multi-organ failure and as per that report, they constitute $5 \%$ of the infected population [11]. Case fatality rate (CFR) is uncertain for SARS-CoV 2 because of a lack of reliable estimate of the total number of cases. It varies from one location to another e.g. $4.4 \%$ in U.S, $4.1 \%$ in Iraq and $14.4 \%$ in Italy [12]. Different demographic variables including the mean age of infected individuals, smoking status, diabetes mellitus, chronic kidney disease, chronic obstructive pulmonary disease (COPD), heart failure (HF) and some other preexisting comorbidities have been implicated to play a significant role in the disparity of disease severity. From China and Italy, the data is suggestive of high case fatality (up to 20\%) among the older population who have 2 or more pre-existing comorbidities [13].

Different laboratory findings including lymphopenia, thrombocytopenia, elevated LDH, d-dimer, elevated liver enzymes, increased prothrombin time, and the elevation of inflammatory markers; CRP and ferritin have been reported with poor outcomes [14].

To mitigate transmission in the community, current public 
health strategies are focusing on breaking the chain of transmission by rapid identification of exposure, isolation of cases and self-quarantine of exposed people. The primary purpose of self-isolation is to prevent the community from the spread of the infection. As per the CDC and WHO guidelines, self-isolation can be discontinued either based on the repeat testing or non-test based strategy. According to a test-based strategy, two nasopharyngeal specimens collected at least 24 hours apart should be negative alongside the resolution of fever and improvement of respiratory symptoms. If a non-test-based strategy is being followed, before discontinuation of self-isolation one must be symptom-free for at least 3 days and the onset of first symptoms should be a week older. In case of mild disease, recovery is expected in two weeks while in severe cases 3-6 weeks is an expected recovery timeline. To date, no medical treatment has shown efficacy in preventing transmission [15].

Inpatient management for severely ill patients involves supportive care, supplemental oxygen via a hi-flow nasal cannula or non-invasive ventilation and, intubation or extracorporeal membrane oxygenation for the persistently hypoxic patients.

Various treatment options including hydroxychloroquine, remdesivir, tocilizumab, and convalescent plasma are used in hospitalized patients, however, safety and efficacy have not yet been established and none of these is FDA approved so far. The CDC panel has recently recommended against the use of hydroxychloroquine and/or azithromycin except in clinical trials. Release of the coronavirus RNA genome into the cytoplasm of the host cell and subsequent viral replication is followed by a cytokine storm or Cytokine Release Syndrome (CRS) leading to life-threatening multiorgan damage. Randomized clinical trials to evaluate the application of cytokine inhibitors e.g. IL-6 inhibitors (Tocilizumab) are under investigation and phase III trials have recently been approved by the U.S. FDA for critically ill patients. The results of another global phase III trial comparing the efficacy of immune modulator tocilizumab plus antiviral agent remdesivir vs placebo plus remdesivir are expected this summer. According to the latest $\mathrm{CDC}$ guidelines, investigational antiviral agent remdesivir can be used in hospitalized patients having $\mathrm{SpO} 2 \leq 94 \%$ on ambient air or those who require supplemental oxygen. A preliminary report of ACTT trials have shown that the use of remdesivir was associated with shorter time to recovery $(\leq 10$ days vs. $>10$ days), however, there was no apparent benefit in mechanically intubated patients. Recent RECOVERY trials showed that dexamethasone reduced 28 days of mortality among COVID-19 hospitalized patients requiring supplemental $\mathrm{O} 2$ or invasive mechanical ventilation. RCT to evaluate the role of convalescent plasma in COVID-19 patients is still underway and most clinical trials are still in the recruiting phase $[16,17]$.

\section{Conclusion}

Increased age ( $>80$ years), hypoxia at the time of presentation and pre-existing co-morbidities are likely related with poor outcomes. Deranged inflammatory markers, ferritin, ESR, d-dimer, IL-6, CRP, LDH, and progressive lymphopenia are possibly related with severity of illness. Management of COVID-19 is mainly supportive. Final results of RECOVERY trial have shown 28 days mortality benefit in patients who received $6 \mathrm{mg} /$ day of dexamethasone for up to 10 days in patients who required invasive mechanical ventilation or supplemental oxygen. Other treatment trials including remdesivir, convalescent plasma, and IL-6 inhibitors are underway.

\section{Author Contributions}

Dr. Mughal and Dr. Kaur has drafted and written the manuscript and shares the common first authorship. Dr. Rehman has collected the radiological data. Dr. Mirza and Dr. Singh has helped with literature review. Dr. Patton and Dr Ghali had revised the final manuscript before submission.

\section{Conflicts of Interest Statement}

I, Mohsin Sheraz Mughal, as a corresponding author declare no conflict of interest on the behalf of all co-authors.

\section{Acknowledgements}

Department of Internal Medicine, Monmouth Medical Center, United States.

\section{References}

[1] The species severe acute respiratory syndrome-related coronavirus: Classifying $2019-\mathrm{nCoV}$ and naming it SARSCov-2. (2020). Nature Microbiology, 5 (4), 536-544. https://doi.org/10.1038/s41564-020-0695-z.

[2] "New Cases of COVID-19 In World Countries." Johns Hopkins Coronavirus Resource Center. Accessed July 10, 2020. https://coronavirus.jhu.edu/data/new-cases.

[3] Verity, R., Okell, L. C., Dorigatti, I., Winskill, P., Whittaker, C., Imai, N., Cuomo-Dannenburg, G., Thompson, H., Walker, P. G., Fu, H., Dighe, A., Griffin, J. T., Baguelin, M., Bhatia, S., Boonyasiri, A., Cori, A., Cucunubá, Z., FitzJohn, R., Gaythorpe, K., Ferguson, N. M. (2020). Estimates of the severity of coronavirus disease 2019: A model-based analysis. The Lancet Infectious Diseases. https://doi.org/10.1016/s14733099(20)30243-7.

[4] Omer, Saad B., Preeti Malani, and Carlos Del Rio. "The COVID-19 Pandemic in the US." JAMA, 2020. doi: 10.1001/jama.2020.5788.

[5] Wang, D., Hu, B., Hu, C., Zhu, F., Liu, X., Zhang, J., Wang, B., Xiang, H., Cheng, Z., Xiong, Y., Zhao, Y., Li, Y., Wang, X., \& Peng, Z. (2020). Clinical Characteristics of 138 Hospitalized Patients With 2019 Novel Coronavirus-Infected Pneumonia in Wuhan, China. JAMA, 323 (11), 1061. https://doi.org/10.1001/jama.2020.1585. 
[6] World Health Organization. Coronavirus disease (COVID-19) technical guidance: Surveillance and case definitions. https://www.who.int/emergencies/diseases/novelcoronavirus2019/technical-guidance/surveillance-and-case-definitions.

[7] Chung, M., Bernheim, A., Mei, X., Zhang, N., Huang, M., Zeng, X., Cui, J., Xu, W., Yang, Y., Fayad, Z. A., Jacobi, A., Li, K., Li, S., \& Shan, H. (2020). CT Imaging Features of 2019 Novel Coronavirus (2019-nCoV). Radiology, 295 (1), 202-207. https://doi.org/10.1148/radiol.2020200230.

[8] Salehi, S., Abedi, A., Balakrishnan, S., \& Gholamrezanezhad, A. (2020). Coronavirus Disease 2019 (COVID-19): A Systematic Review of Imaging Findings in 919 Patients. American Journal of Roentgenology, 1-7. https://doi.org/10.2214/ajr.20.23034.

[9] Xie, X., Zhong, Z., Zhao, W., Zheng, C., Wang, F., \& Liu, J. (2020). Chest CT for Typical 2019-nCoV Pneumonia: Relationship to Negative RT-PCR Testing. Radiology, 200343. https://doi.org/10.1148/radiol.2020200343.

[10] Pan, F., Ye, T., Sun, P., Gui, S., Liang, B., Li, L., Zheng, D., Wang, J., Hesketh, R. L., Yang, L., \& Zheng, C. (2020). Time Course of Lung Changes On Chest CT During Recovery From 2019 Novel Coronavirus (COVID-19) Pneumonia. Radiology, 200370. https://doi.org/10.1148/radiol.202020037.

[11] $\mathrm{Wu}$, Zunyou, and Jennifer M. McGoogan. "Characteristics of and Important Lessons from the Coronavirus Disease 2019 (COVID-19) Outbreak in China." JAMA 323, no. 13 (2020), 1239. doi: 10.1001/jama.2020.2648.
[12] "Mortality Analyses." Johns Hopkins Coronavirus Resource Center. Accessed July 10, 2020. https://coronavirus.jhu.edu/data/mortality.

[13] Onder, G., Rezza, G., \& Brusaferro, S. (2020). Case-fatality rate and characteristics of patients dying in relation to COVID-19 in Italy. https://doi.org/10.1001/jama.2020.4683.

[14] Wu, C., Chen, X., Cai, Y., Xia, J., Zhou, X., Xu, S., Huang, H., Zhang, L., Zhou, X., Du, C., Zhang, Y., Song, J., Wang, S., Chao, Y., Yang, Z., Xu, J., Zhou, X., Chen, D., Xiong, W. Song, Y. (2020). Risk factors associated with acute respiratory distress syndrome and death in patients with coronavirus disease 2019 pneumonia in Wuhan, China. JAMA Internal Medicine. https://doi.org/10.1001/jamainternmed.2020.0994.

[15] Coronavirus disease 2019 (COVID-19). Centers for Disease Control and Prevention. https://www.cdc.gov/coronavirus/2019-ncov/hcp/dispositionin-home-patients.html.

[16] COVID-19 Treatment Guidelines. Last modified May 12, 2020. https://www.covid19treatmentguidelines.nih.gov/.

[17] "The RECOVERY Trial: Dexamethasone for COVID-19?" REBEL EM - Emergency Medicine Blog. Last modified July 3 , 2020. https://rebelem.com/the-recovery-trialdexamethasone-for-covid-19/. 